\title{
Wind Speed Forecasting by Wavelet Neural Networks: A Comparative Study
}

\author{
Chuanan Yao, Xiankun Gao, and Yongchang Yu \\ College of Mechanical and Electrical Engineering, Henan Agricultural University, Zhengzhou 450002, China \\ Correspondence should be addressed to Yongchang Yu; hnyych@163.com
}

Received 30 October 2012; Revised 29 December 2012; Accepted 30 December 2012

Academic Editor: Sheng-yong Chen

Copyright (C) 2013 Chuanan Yao et al. This is an open access article distributed under the Creative Commons Attribution License, which permits unrestricted use, distribution, and reproduction in any medium, provided the original work is properly cited.

\begin{abstract}
Due to the environmental degradation and depletion of conventional energy, much attention has been devoted to wind energy in many countries. The intermittent nature of wind power has had a great impact on power grid security. Accurate forecasting of wind speed plays a vital role in power system stability. This paper presents a comparison of three wavelet neural networks for short-term forecasting of wind speed. The first two combined models are two types of basic combinations of wavelet transform and neural network, namely, compact wavelet neural network (CWNN) and loose wavelet neural network (LWNN) in this study, and the third model is a new hybrid method based on the CWNN and LWNN models. The efficiency of the combined models has been evaluated by using actual wind speed from two test stations in North China. The results show that the forecasting performances of the CWNN and LWNN models are unstable and are affected by the test stations selected; the third model is far more accurate than the other forecasting models in spite of the drawback of lower computational efficiency.
\end{abstract}

\section{Introduction}

Utilization of renewable energy has been paid increasing attention to around the world. Wind energy is one of the most promising energy resources, and wind power generation has been growing rapidly in many countries [1-3]. Wind power generation has rapidly developed over the last decade and global wind power installations reached $240 \mathrm{GW}$ in 2011, accounting for about $5 \%$ of global power installed capacity; global wind power generation will increase to about $450 \mathrm{GW}$ by the year 2020 [4]. However, the randomness of wind in speed and direction leads to the fluctuation of wind power. The large-scale wind power generation integrated in electrical power systems may cause some problems, including power quality, stability, and especially power dispatching [5]. The accurate forecasting of wind speed has an important effect on solving the instability of wind power generation and increasing wind power penetration [6].

Recently, some researchers have proposed a number of methods to forecast wind speed and wind power, which can be classified as physical methods, spatial correlation methods, conventional statistical methods (i.e., persistence method, ARMA), and artificial intelligence methods [714]. In the last two decades, artificial intelligence methods, including artificial neural network (ANN) [15-18], fuzzy logic, support vector machine $[19,20]$, are applied widely in energy engineering area $[21,22]$. Among the methods, ANN is one of the most widely used models and a promising technology in wind speed forecast [3, 15-18].

However, ANN methods have limitations with nonstationary data, which may not be able to handle nonstationary data if the preprocessing of the input data is not done [23]. Wind speed is influenced by many factors, and its change displays a strong stochastic and intermittent feature. A single forecasting model cannot fully approximate the characteristics of wind speed.

Wavelet transform has been applied to a number of disciplines outside of the power system, and it has been found to be very effective to analyze nonstationary time series [24]. Wavelet transform not only has the multiresolution characteristics but also can capture the local feature of signal in both time and frequency domains [25]. The wavelettransformed data can improve the ability of a forecasting 
model by capturing useful information on various resolution levels.

Recently, some hybrid prediction techniques using the combination of wavelet transform and ANN have been developed for wind speed prediction. There are two types of basic combination forms based on wavelet transform and ANN. The first combination method uses discrete wavelet transform (DWT) as a preprocessing tool to decompose the original time series signal into some subsequences with different frequency bands. Then ANN models are used as forecasting modules to predict these subsequences, respectively. The combination method, namely, loose wavelet neural network (LWNN) in this study has been applied in energy and power systems $[26,27]$. The second combination method is similar to back propagation neural networks (BPNN) and uses a kind of wavelet basis function to replace sigmoidal activation function of the hidden layer nodes of BPNN. This combination network is presented firstly by Zhang and Benveniste [28], namely, compact wavelet neural network (CWNN) in this study. At present, this method has been widely applied to various fields $[29,30]$. Other combination methods are rarely reported in the literature [31-35].

In this study, we are interested in combined models based on the wavelet transform and neural network for wind speed forecasting. This paper presents three types of short-term wind speed forecasting models, including the preceding two wavelet neural networks (CWNN and LWNN). The third model is a new hybrid method based on a combination of the preceding two types of wavelet neural networks. The aim of this paper is to investigate the three models and compare the forecasting performance for wind speed by using the actual wind speed data in two different wind farms.

The remainder parts of the paper are organized as follows. In Section 2, three models based on the combination of wavelet and neural network are outlined. In Section 3, the model parameters are described, and some evaluating indexes used in this study are presented. In Section 4, obtained numerical results and discussion from these methods are presented. In Section 5, some relevant conclusions about the performance achieved by comparing CWNN, LWNN, and LCWNN models are provided.

\section{Three Types of Wavelet Neural Network Models}

2.1. The CWNN Model. The architecture of the CWNN model is based on the topology of BPNN. In this method, a kind of continuous wavelet basis function (i.e., Morlet wavelet or Mexican hat wavelet) is employed to replace sigmoidal activation function of the hidden layer nodes, and the scaling and shifting parameters of wavelet basis functions are used to replace the corresponding connection weights and thresholds from the input to the hidden layer. Wavelet function is a local function and influences the output of the hidden layer only in some local range [36]. The CWNN method may solve the conventional problems of poor convergence or trapping in local optima encountered in BPNN. The structure of the CWNN model is shown in Figure 1(a).
In Figure $1(\mathrm{a}), x_{1}, x_{2}, \ldots, x_{k}$ and $y_{1}, y_{2}, \ldots, y_{m}$ are the input and the output variables of the model, respectively; $\omega_{i j}$ is the weight for the connection between the $i$ th node of the input layer and the $j$ th node of the hidden layer; $\omega_{j k}$ is the weight for the connection between the $j$ th node of the hidden layer and the $k$ th node of the output layer. The output of each hidden layer neuron is calculated with the following formula:

$$
h(j)=\psi_{j}\left(\frac{\sum_{i=1}^{k} \omega_{i j} x_{i}-b_{j}}{a_{j}}\right), \quad j=1,2, \ldots, l,
$$

where $h(j)$ is the output of the $j$ th node in the hidden layer; $\psi_{j}$ denotes wavelet basis function of the $j$ th node in the hidden layer; $a_{j}$ and $b_{j}$ are the scaling and shifting parameters of wavelet function; $l$ defines the number of hidden layer nodes.

The output of the network is given by

$$
y_{k}=\sum_{i=1}^{l} \omega_{i k} h(i), \quad k=1,2, \ldots, m,
$$

where $\omega_{i k}$ is the weight for the connection between the hidden and the output layer. $h(i)$ is the output of the $i$ th node in hidden layer. $m$ is the number of the output layer nodes.

Similar to the training algorithm of BPNN, the model adopts gradient descent rule to correct the connection weights and the parameters of wavelet basis function.

2.2. The LWNN and LCWNN Models. Both of the LWNN and LCWNN models consist of two parts: the preprocessing module based on discrete wavelet transform and the prediction module based on neural network.

Figures 1(b) and 1(c) show the architecture of the LWNN and LCWNN models, respectively. Their structures are similar. In the preprocessing modules, both models take discrete wavelet transform as preprocessing tools to decompose original wind speed time series $x(n)$ into a set of wavelet coefficients, then reconstruct these coefficients into the time subseries. In the prediction modules, both models take neural networks as forecasting methods to predict the subseries. However, the difference between the two prediction modules is that the LWNN model uses the conversional BP neural networks to forecast the decomposed subseries, and the LCWNN model uses the CWNN model to predict them. In Figures 1(b) and 1(c), $c a_{j}, c d_{j}, c d_{j-1}, \ldots, c d_{1}(j$ is the scaling factor) are wavelet coefficients and $a_{j}(n), d_{j}(n)$, $d_{j-1}(n), \ldots, d_{1}(n)$ are the reconstructed time subseries. $c a_{i}$ and $a_{i}(n)$ denote low-frequency component or approximation of original wind speed signal; $d_{j}(n), d_{j-1}(n), \ldots, d_{1}(n)$ and $d_{i}(n)$ represent high-frequency components or details of original wind speed signal. follows:

The modeling steps of the two methods are described as

(1) Use discrete wavelet transform to decompose original time series $x(n)$ into a set of wavelet coefficients at scaling factor $j$, including low-frequency coefficients, $j$ high-frequency coefficients, and then separately reconstruct these coefficient series into a set of time subseries that is equal to the length of original wind speed time series. 


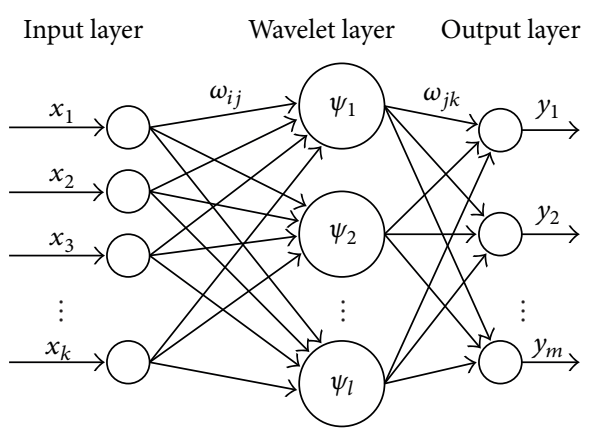

(a) CWNN model

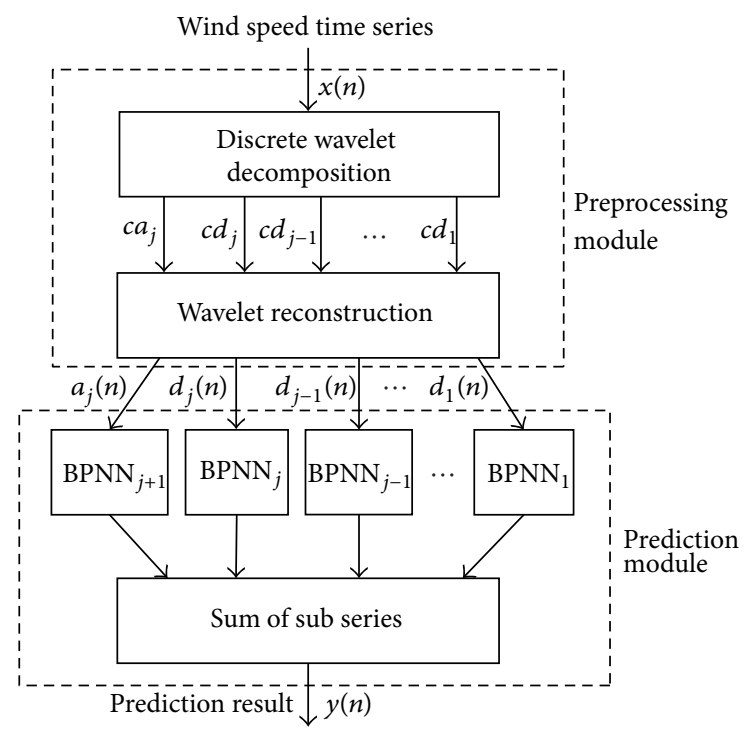

(b) LWNN model

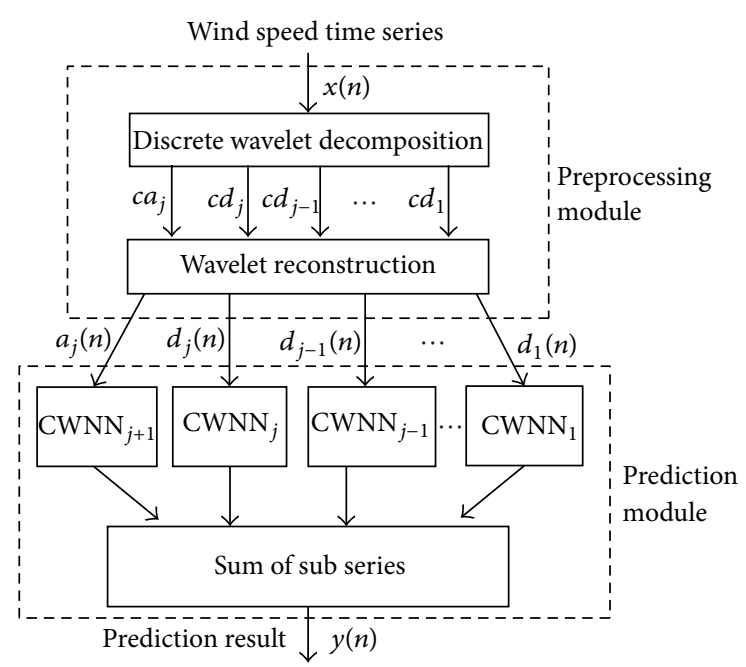

(c) LCWNN model

FIGURE 1: Structures of the three types of wavelet neural network models.
(2) Establish the BPNN and CWNN prediction models for these subseries, respectively, and make the shortterm prediction for each subseries.

(3) Calculate the sum of forecasting results of all subseries to obtain the final forecasting for original time series.

\section{Wind Speed Prediction Based on the Three Models}

3.1. Wind Speed Data. Two stations (test station 1 and test station 2) located in Inner Mongolia Autonomous Region of China are used in this study. The hourly wind speed data are recorded from two wind farms in March 2010. Monitors on site sampled the wind speed data for every five-minute interval, which are collected 12 times in an hour. These measured data have been averaged to obtain the hourly mean wind speed (720 samplings). These samplings are divided into two groups. The first group of the samplings (1st-600th) is used for training and establishing models, and the second group of the samplings (601st-720th) is used for testing the validity of the prediction models.

3.2. Forecasting Model Parameters. For purpose of analyzing and assessing the forecasting performances of the combined models, two sets of wind speed data in two test stations are input into CWNN, LWNN, LCWNN, and the conventional BPNN models, respectively.

Wavelet transform is used in the LCWNN model as well as the CWNN and LWNN models. In the LWNN and LCWNN models, their preprocessing modules both apply discrete wavelet transform, and the wind speed series of test station 1 is decomposed discretely using db4 (Daubechies wavelet family) at scaling factor 3 , respectively; the wind speed series in test station 2 is decomposed discretely using $\mathrm{db} 3$ at scaling factor 2 , respectively.

In the forecasting modules of CWNN and LCWNN for every test station, the transfer functions both apply continuous Morlet mother wavelet function. The neurons in the input and the hidden layer in the forecasting modules are chosen by trial and error. The model parameters of the three wavelet neural networks and BP network with a single algorithm are shown in Table 1 .

3.3. Evaluation Criteria. To compare the efficiency of the three wavelet neural networks for the prediction of wind speed, some error criteria are employed in this study. The mean absolute error (MAE), the root mean squared error (RMSE), and the mean absolute percentage error (MAPE) are defined as follows:

$$
\begin{aligned}
\text { MAE } & =\frac{1}{n} \sum_{i=1}^{n}\left|\hat{y}_{i}-y_{i}\right|, \\
\text { RMSE } & =\sqrt{\frac{1}{n} \sum_{i=1}^{n}\left(\hat{y}_{i}-y_{i}\right)^{2},} \\
\text { MAPE } & =\frac{100}{n} \sum_{i=1}^{n}\left|\frac{\hat{y}_{i}-y_{i}}{y_{i}}\right|,
\end{aligned}
$$


TABLE 1: Model parameters of the three wavelet neural networks and BP networks.

\begin{tabular}{|c|c|c|c|c|c|c|c|}
\hline \multirow{2}{*}{ Test station } & \multirow{2}{*}{ Model } & \multicolumn{2}{|c|}{ Wavelet preprocessing module } & \multicolumn{4}{|c|}{ Neural network prediction module } \\
\hline & & $\begin{array}{l}\text { Wavelet } \\
\text { function }\end{array}$ & $\begin{array}{l}\text { Decomposition } \\
\text { coefficients }\end{array}$ & $\begin{array}{c}\text { Structure of } \\
\text { network }\end{array}$ & $\begin{array}{l}\text { Transfer function of } \\
\text { hidden layer }\end{array}$ & $\begin{array}{c}\text { Transfer function of } \\
\text { output layer }\end{array}$ & $\begin{array}{l}\text { Training } \\
\text { algorithm }\end{array}$ \\
\hline \multirow{10}{*}{ Test station 1} & BPNN & \multirow{5}{*}{$\mathrm{db} 4$} & \multirow{5}{*}{$c a_{3}, c d_{3}, c d_{2}, c d_{1}$} & $4-12-1^{*}$ & tansig & purelin & trainlm \\
\hline & CWNN & & & $4-9-1$ & Morlet & purelin & trainlm \\
\hline & \multirow{4}{*}{ LWNN } & & & $c a_{3}: 3-11-1$ & tansig & purelin & trainlm \\
\hline & & & & $c d_{3}: 4-7-1$ & tansig & purelin & trainlm \\
\hline & & & & $c d_{2}: 4-12-1$ & tansig & purelin & trainlm \\
\hline & & \multirow{5}{*}{$\mathrm{db} 4$} & \multirow{5}{*}{$c a_{3}, c d_{3}, c d_{2}, c d_{1}$} & $c d_{1}: 4-12-1$ & tansig & purelin & trainlm \\
\hline & \multirow{4}{*}{ LCWNN } & & & $c a_{3}: 3-9-1$ & tansig & purelin & trainlm \\
\hline & & & & $c d_{3}: 4-7-1$ & tansig & purelin & trainlm \\
\hline & & & & $c d_{2}: 4-7-1$ & tansig & purelin & trainlm \\
\hline & & & & $c d_{1}: 4-12-1$ & tansig & purelin & trainlm \\
\hline \multirow{8}{*}{ Test station 2} & BPNN & & & 4-11-1 & tansig & purelin & trainlm \\
\hline & CWNN & & & $3-8-1$ & Morlet & purelin & trainlm \\
\hline & & & & $c a_{2}: 3-8-1$ & tansig & purelin & trainlm \\
\hline & LWNN & $\mathrm{db} 3$ & $c a_{2}, c d_{2}, c d_{1}$ & $c d_{2}: 4-7-1$ & tansig & purelin & trainlm \\
\hline & & & & $c d_{1}: 4-12-1$ & tansig & purelin & trainlm \\
\hline & & & & $c a_{2}: 3-9-1$ & tansig & purelin & trainlm \\
\hline & LCWNN & $\mathrm{db} 3$ & $c a_{2}, c d_{2}, c d_{1}$ & $c d_{2}: 3-10-1$ & tansig & purelin & trainlm \\
\hline & & & & $c d_{1}: 4-11-1$ & tansig & purelin & trainlm \\
\hline
\end{tabular}

${ }^{*} m$-n-p denotes structure pattern of neural network. $m, n, p$ are the numbers of the input, the hidden, and the output layer nodes, respectively.

TABLE 2: MAE, RMSE, and MAPE by deferent models for test station 1.

\begin{tabular}{lcccc}
\hline Model & $\begin{array}{c}\text { MAE } \\
(\mathrm{m} / \mathrm{s})\end{array}$ & $\begin{array}{c}\text { RMSE } \\
(\mathrm{m} / \mathrm{s})\end{array}$ & $\begin{array}{c}\text { MAPE } \\
(\%)\end{array}$ & $\begin{array}{c}\text { Average computation } \\
\text { time }(\mathrm{s})\end{array}$ \\
\hline Persistence & 1.279 & 1.432 & 36.65 & 0.12 \\
BPNN & 1.186 & 1.338 & 30.19 & 3.36 \\
CWNN & 1.015 & 1.296 & 29.44 & 5.73 \\
LWNN & 0.475 & 0.522 & 13.17 & 9.69 \\
LCWNN & 0.426 & 0.468 & 11.82 & 19.74 \\
\hline
\end{tabular}

where $n$ is the number of testing wind speed values; $y_{i}$ and $\widehat{y}_{i}$ are the actual and predicted wind speed at time $i$, respectively.

\section{Comparative Analysis}

In the study, the persistence method and the conventional BPNN model are adopted as the benchmark for testing the performances of the three wavelet neural networks. These models are used for forecasting wind speed in two wind farms. Figure 2 and Tables 2 and 3 are the forecasting results for these prediction models.

Figures 2(a) and 2(b) show the forecasting results of wind speed made in two test stations, respectively. The subfigures, respectively, show the measured and the forecasted wind speed values obtained by the persistence method, BPNN, CWNN, LWNN, and LCNN.
TABLE 3: MAE, RMSE, and MAPE by deferent models for test station 2 .

\begin{tabular}{lcccc}
\hline Model & $\begin{array}{c}\text { MAE } \\
(\mathrm{m} / \mathrm{s})\end{array}$ & $\begin{array}{c}\text { RMSE } \\
(\mathrm{m} / \mathrm{s})\end{array}$ & $\begin{array}{c}\text { MAPE } \\
(\%)\end{array}$ & $\begin{array}{c}\text { Average computation } \\
\text { time }(\mathrm{s})\end{array}$ \\
\hline Persistence & 1.382 & 1.593 & 16.71 & 0.10 \\
BPNN & 1.343 & 1.584 & 16.26 & 3.49 \\
CWNN & 0.879 & 0.984 & 10.65 & 6.26 \\
LWNN & 0.808 & 0.958 & 9.78 & 11.70 \\
LCWNN & 0.549 & 0.621 & 6.64 & 22.35 \\
\hline
\end{tabular}

Tables 2 and 3 give the comparative MAE, RMSE and MAPE error results obtained by the three types of wavelet network models (CWNN, LWNN and LCWNN) along with the persistence method and BPNN according to the error criteria in (3). The computation time of each algorithm is also shown in Tables 2 and 3.

Table 2 presents the results in test station 1 . It can be seen that the MSE, RMSE, and MAPE values for the three combination wavelet neural network models are all smaller than those obtained by the persistence method and BPNN. For example, the MSE values for the CWNN, LWNN, and LCWNN are $1.015 \mathrm{~m} / \mathrm{s}, 0.475 \mathrm{~m} / \mathrm{s}$, and $0.426 \mathrm{~m} / \mathrm{s}$, respectively, which are reduced by $20.6 \%, 62.9 \%$, and $66.7 \%$ compared with those of the persistence method, and $14.4 \%, 59.9 \%$, and $64.1 \%$ compared with those of BPNN. Among the three combination models, the LCWNN method presents better forecasting accuracy. The MAE, RMSE, and MAPE values 

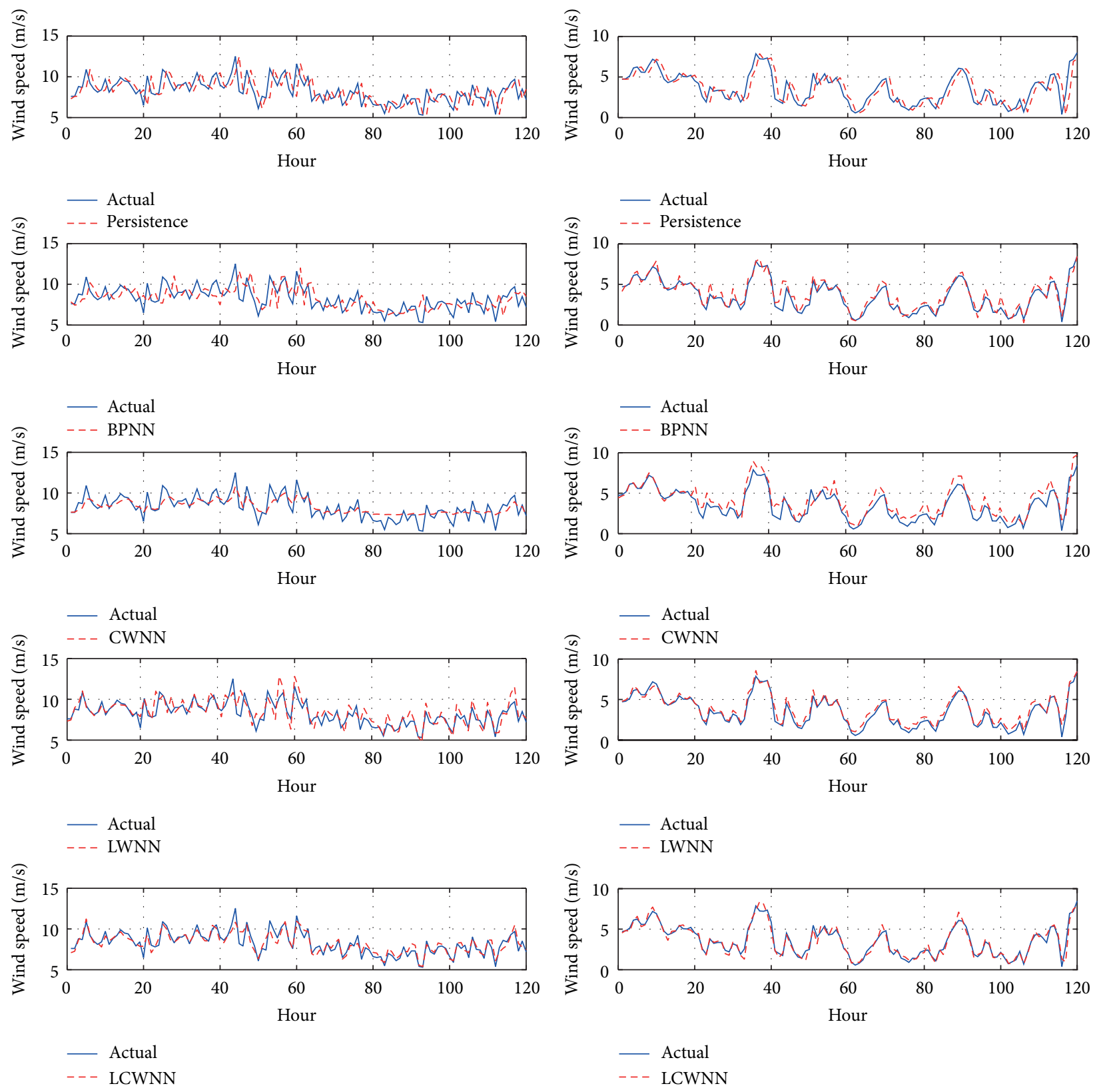

(a) for test station 1

(b) for test station 2

FIGURE 2: Forecasting results of wind speed series made by different models.

of the LCWNN model are $0.426 \mathrm{~m} / \mathrm{s}, 0.468 \mathrm{~m} / \mathrm{s}$, and $11.82 \%$, respectively. Improvements in the errors of the LCWNN model as against the CWNN model are 49.7\%, 63.9\%, and $59.9 \%$. But the errors of the LWNN model are close to those of the LCWNN model.

Table 3 also lists the simulation results where these forecasting models are applied in test station 2 . Table 3 shows that the prediction accuracy of the three wavelet neural network models also outperforms that of the persistence method and BPNN. But for the wavelet neural network models, the prediction accuracy of CWNN are close to that of LWNN, and both are much lower than that of the LCWW method.
From Tables 2 and 3, the error values of the LCWNN model are the smallest in two test stations. In test station 1 , the error values of CWNN are worse than those of LWNN; whereas in test station 2, the comparison between CWNN and LWNN shows that the two models yield similar results, and it is difficult to definitively conclude which model is better.

Besides, it can also be observed that the prediction performance of the persistence method is the weakest compared with the other four models based on neural networks. The persistence method assumes the current measured value as the prediction value of the next wind speed, and its 
forecasting accuracy depends on the similarity of adjacent wind speed data. Some research shows the models based on neural networks generally outperform the persistence method [18].

Additionally, simulation results also exhibit that the prediction performance of BPNN model is weaker than the other three models based on the combination of wavelet transform and neural network, which can be explained by the fact that BP algorithm is not a global optimization method and easy to fall into local optimum, and its approximation and generalization abilities relay on the learning sample selected. The prediction accuracy can be improved by CWNN, LWNN, and LCWNN approaches because the wavelet transform can produce a good local representation of wind speed signals in both time and frequency domains. However, the prediction accuracy by CWNN and LWNN is unstable, easily influenced by geographical conditions, and the LCWNN model based on the combination of CWNN and LWNN can capture the complex features of wind speed and overcome the instability occurred in CWNN and LWNN models.

Unfortunately, the wavelet neural network methods spend more computation time than the persistence method and BPNN. Among the combined methods, the computational efficiency of the LWNN and the LCWNN methods is lower than CWNN and BPNN, because the prediction modules of the two methods both consist of several other models (i.e., BPNN and CWNN) in Figure 1. As shown in Tables 2 and 3, the average computation time of LCWNN is much longer than the other models using MATLAB on a PC with $2 \mathrm{~GB}$ of RAM and a $2.8 \mathrm{GHz}$ based processor.

\section{Conclusions}

In this paper, three types of forecasting methods for wind speed based on the combinations of wavelet transform and neural networks were proposed. These combined methods were investigated by using two sets of wind speed data from different test stations in North China and compared with the persistence method and the conventional single BPNN. Due to the ability of wavelet transform for revealing the local characteristics of nonlinear time series and of neural network for self-learning and nonlinear approximation properties, the three wavelet neural network models have better forecasting accuracy than the persistence method and BPNN. The comparative result of different wavelet neural networks shows that the CWNN and the LCNN methods are unstable, easily influenced by different wind data sets; the LCWNN method combines the advantages of the CWNN and the LCWW models, and can reduce the prediction errors and improve the uncertainty. However, the operation time of the LCWNN method is much longer than other models because its prediction module consists of several CWNN models. In spite of its lower computational efficiency, it can be recommended that the LCWNN model could be a robust method for wind speed forecasting.

\section{Acknowledgments}

The research was supported in part by Science and Technology Development entitled "Research on remote monitoring technology of hybrid wind-photovoltaic system" from the Department of Science and Technology in Henan province of China (Grant no. 122102210128) and the Key Laboratory of Zhengzhou for agricultural testing technology and instrumentation (Grant no. 121PYFZX184).

\section{References}

[1] O. Carranza, E. Figueres, G. Garcerá, and L. G. Gonzalez, "Comparative study of speed estimators with highly noisy measurement signals for Wind Energy Generation Systems," Applied Energy, vol. 88, no. 3, pp. 805-813, 2011.

[2] J. Zhao, X. Li, J. Hao, and J. Lu, "Reactive power control of wind farm made up with doubly fed induction generators in distribution system," Electric Power Systems Research, vol. 80, no. 6, pp. 698-706, 2010.

[3] L. Ma, S. Luan, C. Jiang, H. Liu, and Y. Zhang, "A review on the forecasting of wind speed and generated power," Renewable and Sustainable Energy Reviews, vol. 13, no. 4, pp. 915-920, 2009.

[4] J. Li, China Wind Power Outlook 2012, China Environmental Science Press, Beijing, China, 2012.

[5] E. Cadenas and W. Rivera, "Wind speed forecasting in three different regions of Mexico, using a hybrid ARIMA-ANN model," Renewable Energy, vol. 35, no. 12, pp. 2732-2738, 2010.

[6] L. Cao and R. Li, "Short-term wind speed forecasting model for wind farm based on wavelet decomposition," in Proceedings of the 3rd International Conference on Deregulation and Restructuring and Power Technologies (DRPT '08), pp. 2525-2529, April 2008.

[7] S. Y. Chen, C. Y. Yao, G. Xiao, Y. S. Ying, and W. L. Wang, "Fault detection and prediction of clocks and timers based on computer audition and probabilistic neural networks," Lecture Notes on Computer Science, vol. 3512, pp. 952-959, 2005.

[8] S. Castellucci and M. Carlini, "Modelling and simulation for energy production parametric dependence in greenhouses," Mathematical Problems in Engineering, vol. 2010, Article ID 590943, 28 pages, 2010.

[9] H. Liu, H. Q. Tian, C. Chen, and Y. F. Li, "A hybrid statistical method to predict wind speed and wind power," Renewable Energy, vol. 35, no. 8, pp. 1857-1861, 2010.

[10] L. Landberg, "Short-term prediction of the power production from wind farms," Journal of Wind Engineering and Industrial Aerodynamics, vol. 80, no. 1-2, pp. 207-220, 1999.

[11] I. J. Ramirez-Rosado, L. A. Fernandez-Jimenez, C. Monteiro, J. Sousa, and R. Bessa, "Comparison of two new short-term windpower forecasting systems," Renewable Energy, vol. 34, no. 7, pp. 1848-1854, 2009.

[12] S. Chen, Y. Zheng, C. Cattani, and W. Wang, "Modeling of biological intelligence for SCM system optimization," Computational and Mathematical Methods in Medicine, vol. 2012, Article ID 769702, 10 pages, 2012.

[13] C. Cattani, "Shannon wavelets for the solution of integrodifferential equations," Mathematical Problems in Engineering, vol. 2012, Article ID 408418, 22 pages, 2010.

[14] Y. K. Wu and J. S. Hong, "A literature review of wind forecasting technology in the world," in Proceedings of the IEEE Lausanne PowerTech, pp. 504-509, July 2007. 
[15] M. Bilgili, B. Sahin, and A. Yasar, "Application of artificial neural networks for the wind speed prediction of target station using reference stations data," Renewable Energy, vol. 32, no. 14, pp. 2350-2360, 2007.

[16] G. Li and J. Shi, "On comparing three artificial neural networks for wind speed forecasting," Applied Energy, vol. 87, no. 7, pp. 2313-2320, 2010.

[17] M. Monfared, H. Rastegar, and H. M. Kojabadi, "A new strategy for wind speed forecasting using artificial intelligent methods," Renewable Energy, vol. 34, no. 3, pp. 845-848, 2009.

[18] G. Li, J. Shi, and J. Zhou, "Bayesian adaptive combination of short-term wind speed forecasts from neural network models," Renewable Energy, vol. 36, no. 1, pp. 352-359, 2011.

[19] M. A. Mohandes, T. O. Halawani, S. Rehman, and A. A. Hussain, "Support vector machines for wind speed prediction," Renewable Energy, vol. 29, no. 6, pp. 939-947, 2004.

[20] J. Zhou, J. Shi, and G. Li, "Fine tuning support vector machines for short-term wind speed forecasting," Energy Conversion and Management, vol. 52, no. 4, pp. 1990-1998, 2011.

[21] H. Bouzgou and N. Benoudjit, "Multiple architecture system for wind speed prediction," Applied Energy, vol. 88, no. 7, pp. 24632471, 2011.

[22] S. A. P. Kani and M. M. Ardehali, "Very short-term wind speed prediction: a new artificial neural network-Markov chain model," Energy Conversion and Management, vol. 52, no. 1, pp. 738-745, 2011.

[23] J. Adamowski and K. Sun, "Development of a coupled wavelet transform and neural network method for flow forecasting of non-perennial rivers in semi-arid watersheds," Journal of Hydrology, vol. 390, no. 1-2, pp. 85-91, 2010.

[24] J. Eynard, S. Grieu, and M. Polit, "Wavelet-based multiresolution analysis and artificial neural networks for forecasting temperature and thermal power consumption," Engineering Applications of Artificial Intelligence, vol. 24, no. 3, pp. 501-516, 2011.

[25] N. Amjady and F. Keynia, "Short-term load forecasting of power systems by combination of wavelet transform and neuroevolutionary algorithm," Energy, vol. 34, no. 1, pp. 46-57, 2009.

[26] J. P. S. Catalão, H. M. I. Pousinho, and V. M. F. Mendes, "Shortterm wind power forecasting in Portugal by neural networks and wavelet transform," Renewable Energy, vol. 36, no. 4, pp. 1245-1251, 2011.

[27] S. J. Yao, Y. H. Song, L. Z. Zhang, and X. Y. Cheng, "Wavelet transform and neural networks for short-term electrical load forecasting," Energy Conversion and Management, vol. 41, no. 18, pp. 1975-1988, 2000.

[28] Q. Zhang and A. Benveniste, "Wavelet networks," IEEE Transactions on Neural Networks, vol. 3, no. 6, pp. 889-898, 1992.

[29] A. Mellit, M. Benghanem, and S. A. Kalogirou, "An adaptive wavelet-network model for forecasting daily total solarradiation," Applied Energy, vol. 83, no. 7, pp. 705-722, 2006.

[30] Y. Jin, G. Chen, and H. Liu, "Fault diagnosis of analog circuit based on wavelet neural network," Chinese Journal of Scientific Instrument, vol. 28, no. 9, pp. 1600-1604, 2007 (Chinese).

[31] C. Cattani, S. Chen, and G. Aldashev, "Information and modeling in complexity," Mathematical Problems in Engineering, vol. 2012, Article ID 868413, 4 pages, 2012.

[32] S. Y. Chen, "Kalman filter for robot vision: a survey," IEEE Transactions on Industrial Electronics, vol. 59, no. 11, pp. 44094420, 2012.
[33] M. Li, S. C. Lim, and S. Chen, "Exact solution of impulse response to a class of fractional oscillators and its stability," Mathematical Problems in Engineering, vol. 2011, Article ID 657839, 9 pages, 2011.

[34] S. Y. Chen, H. Tong, and C. Cattani, "Markov models for image labeling," Mathematical Problems in Engineering, vol. 2012, Article ID 814356, 18 pages, 2012.

[35] W. Huang and S. Chen, "Epidemic metapopulation model with traffic routing in scale-free networks," Journal of Statistical Mechanics, vol. 2011, no. 12, Article ID P12004, 19 pages, 2011.

[36] A. Mellit, "Artificial Intelligence technique for modeling and forecasting of solar radiation data: a review," International Journal of Artificial Intelligence and Soft Computing, vol. 1, no. 1, pp. 52-76, 2008. 


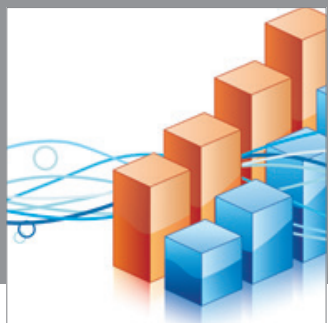

Advances in

Operations Research

mansans

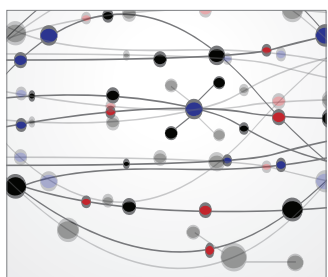

The Scientific World Journal
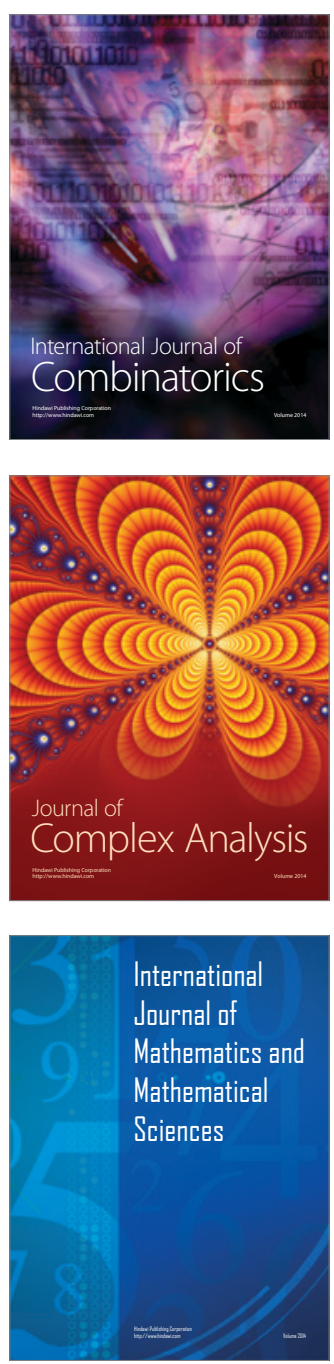
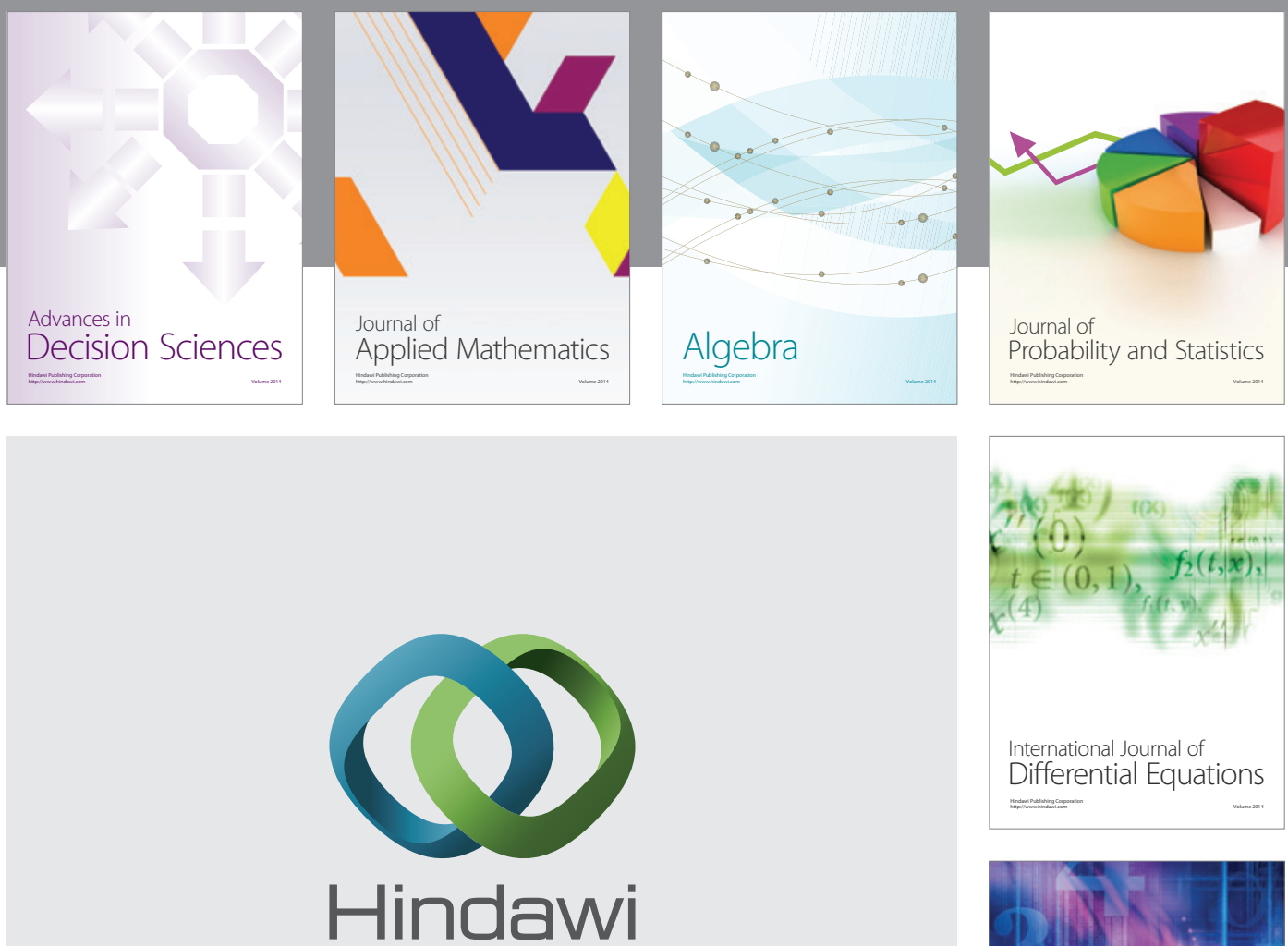

Submit your manuscripts at http://www.hindawi.com
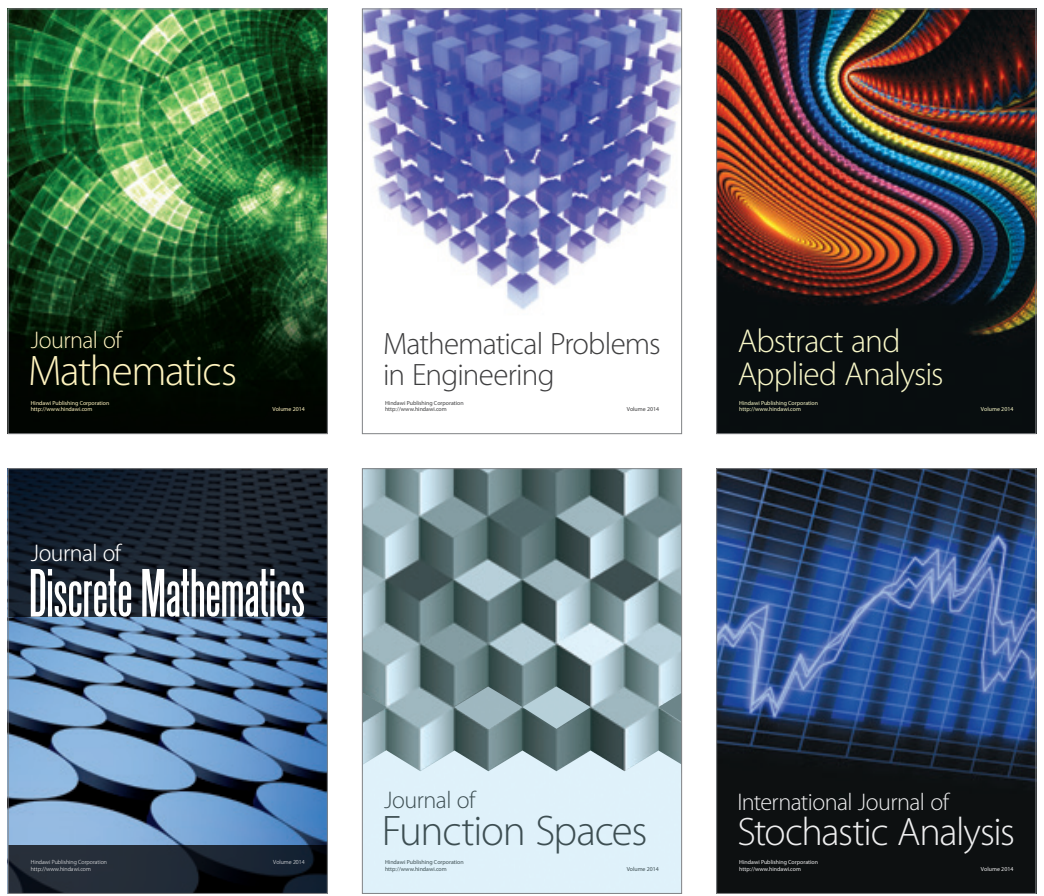

Journal of

Function Spaces

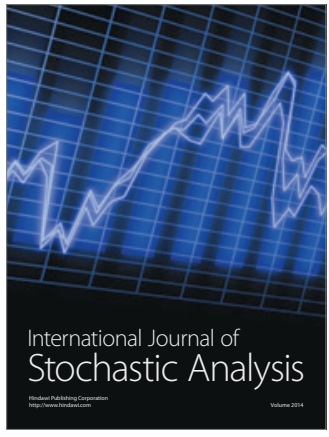

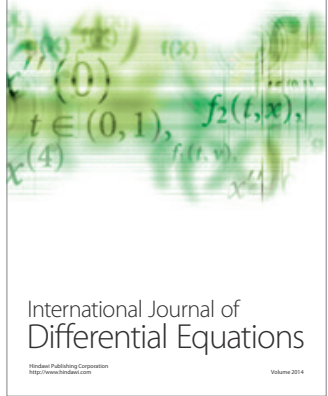
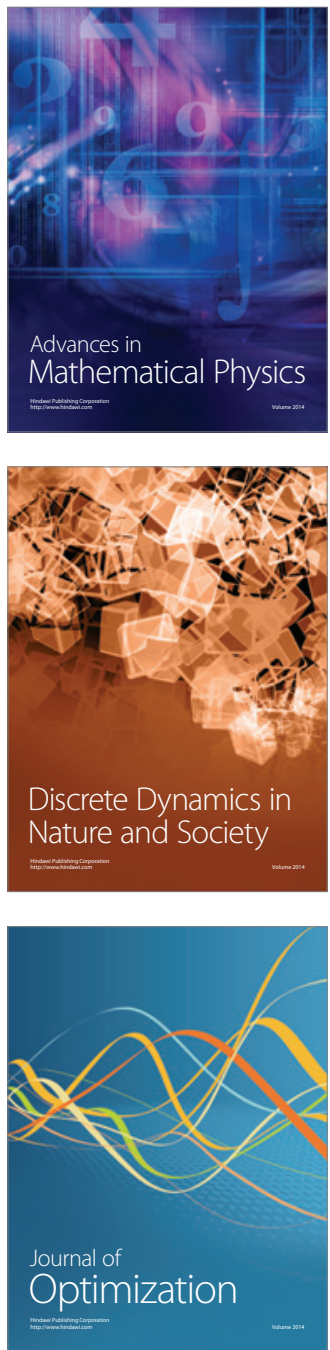